\title{
Sculpting organ innervation
}

\author{
Barbara L. Hempstead
}

Department of Medicine, Weill Medical College of Cornell University, New York, New York, USA.

\begin{abstract}
Neurotrophic growth factors, including nerve growth factor (NGF) and glialderived neurotrophic factor (GDNF), have well-established roles in promoting the innervation of target tissues, yet little is known about how the temporal and organ-specific expression of these factors is regulated. A new study (see the related article beginning on page 876) reveals that NGF is a direct target of the well-characterized peptide factor endothelin-1 (ET-1), and that ET-1induced NGF expression is required for sympathetic innervation of the developing heart. These results, and recent studies implicating GDNF and ET-3 in the patterning of the enteric nervous system, suggest that specific pairing of endothelins and neurotrophic factors may be used in distinct target organs to coordinate neuronal migration, differentiation, and survival.
\end{abstract}

The development of the neural crestderived sensory, autonomic, and enteric nervous systems is an intricate process that directs the egress of crest cells from the dorsal neural tube, their migration and differentiation in distinct ganglia, and their ultimate projections to maturing peripheral organs. This feat requires the coordination of complex sets of target-derived cues that play instructive roles in neuronal differentiation, survival, and pathfinding in vivo, to ensure that neuron numbers precisely match target innervation requirements. Although neurotrophic factors, including the neurotrophins nerve growth factor (NGF), brain-derived neurotrophic factor (BDNF), and neurotrophin-3 (NT-3), as well as glial-derived neurotrophic factor (GDNF), are well-characterized targetderived factors that promote the survival and differentiation of peripheral neurons (1), little is known about the upstream mechanisms that regulate the temporal and spatial expression of these factors to dictate organ-specific patterns of axon guidance, aside from the regulation of NT- 3 in mesenchyme by Wnt factors (2). In the heart, myocyte-derived NGF critically regulates sympathetic innervation (3), an event that occurs in late gestation. In this issue of the $J C I$, Ieda and colleagues (4) uncover a critical role for endothelin-1 (ET-1), a member

Nonstandard abbreviations used: brain-derived neurotrophic factor (BDNF); endothelin (ET); glial-derived neurotrophic factor (GDNF); nerve growth factor (NGF); neurotrophin-3 (NT-3).

Conflict of interest: The author has declared that no conflict of interest exists.

Citation for this article:

J. Clin. Invest. 113:811-813 (2004).

doi:10.1172/JCI200421309. of the endothelin family of peptides that have numerous roles in cardiovascular development, in regulating NGF expression in the heart. The endothelins are a family of 21-amino acid peptides originally characterized by their potent vasopressive effects. Enzymatically cleaved from a preproform by the sequential actions of endothelin-converting enzymes, ET- 1 is the best-characterized member in the cardiovascular system and binds to two distinct seven-transmembrane $\mathrm{G}$ protein-coupled receptors, $\mathrm{ET}_{\mathrm{A}}$ and $\mathrm{ET}_{\mathrm{B}}$ (5). Prominent effects of endothelin signaling in cardiovascular development include the promotion of Purkinje fiber differentiation (6), craniofacial and aortic arch patterning via actions on cranial and cardiac neural crest (7), and cardiac outflow tract remodeling (8). In many regards, symptoms observed in mice deficient in ET-1, endothelin-converting enzyme-1, or $\mathrm{ET}_{\mathrm{A}}$ resemble those associated with human congenital cardiovascular malformations such as CATCH 22 syndrome, although only a few downstream targets of ET-1, including heart and neural crest derivatives (known as HANDs) and Goosecoid, have been characterized $(8,9)$.

In the current study, Ieda and colleagues document that ET-1, but not several other growth factors known to act on embryonic cardiomyocytes, upregulates NGF mRNA and protein expression in myocyte cultures

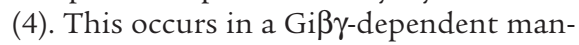
ner, consistent with the known expression of $\mathrm{ET}_{\mathrm{A}}$ by cardiac myocytes, and by established intracellular signaling pathways downstream of the $\mathrm{ET}_{\mathrm{A}}$ receptor. Using ET-1 gene-targeted mice, the authors demonstrate that ET-1 markedly induces NGF expression by cardiac myocytes between embryonic days 15.5 and 18.5 , when stellate ganglion sympathetic neurons are extending their projections to the heart. In the absence of ET-1, fiber density is markedly diminished, cardiac norepinephrine levels fail to increase, and stellate ganglion neurons die. These results are consistent with recent studies of Glenbova and Ginty (10) using mice doubly deficient in both Bax, to impair neuronal apoptosis, and NGF. Their studies indicate that although NGF is not required for the formation of the stellate ganglion, it is required to promote extensive fiber growth to innervate coronary arteries and cardiac myocytes. Ieda and colleagues use genetic rescue experiments, crossing ET-1null mice with transgenic mice overexpressing NGF in cardiac myocytes, to confirm that myocyte-derived NGF can restore sympathetic innervation despite the loss of ET-1 (4). Although the in vivo source of ET-1 in the late-gestational heart is not documented, these complementary approaches provide convincing evidence that endothelin receptor signaling in myocytes is required for optimal NGF induction and subsequent target innervation.

\section{Evidence of endothelin-neurotrophic factor pairing to establish the innervation of other organs}

In contrast to the relatively uncomplicated neural inputs to the heart, the enteric nervous system is composed of at least 14 different types of neurons that reside within the gut wall to control intestinal motility, regulate blood flow and intestinal secretions, and respond to stimuli from the gut lumen (11). Important genetic clues from patients with distal aganglionosis, also known as Hirschsprung disease, have pointed to the critical role of GDNF and its Ret receptor in establishing the normal enteric plexus. Gene targeting of GDNF, Ret, or, interestingly, ET-3 or its $\mathrm{ET}_{\mathrm{B}}$ receptor results in a similar phenotype of colonic aganglionosis; indeed, patients with Hirschsprung disease have mutations in Ret or $\mathrm{ET}_{\mathrm{B}}$ at incidences approaching $50 \%$ or $5 \%$, respectively $(11,12)$. These observations suggest that GDNF and ET-3 may interact to promote the survival of enteric neurons, as well as the growth of distal processes into the developing colon wall. 


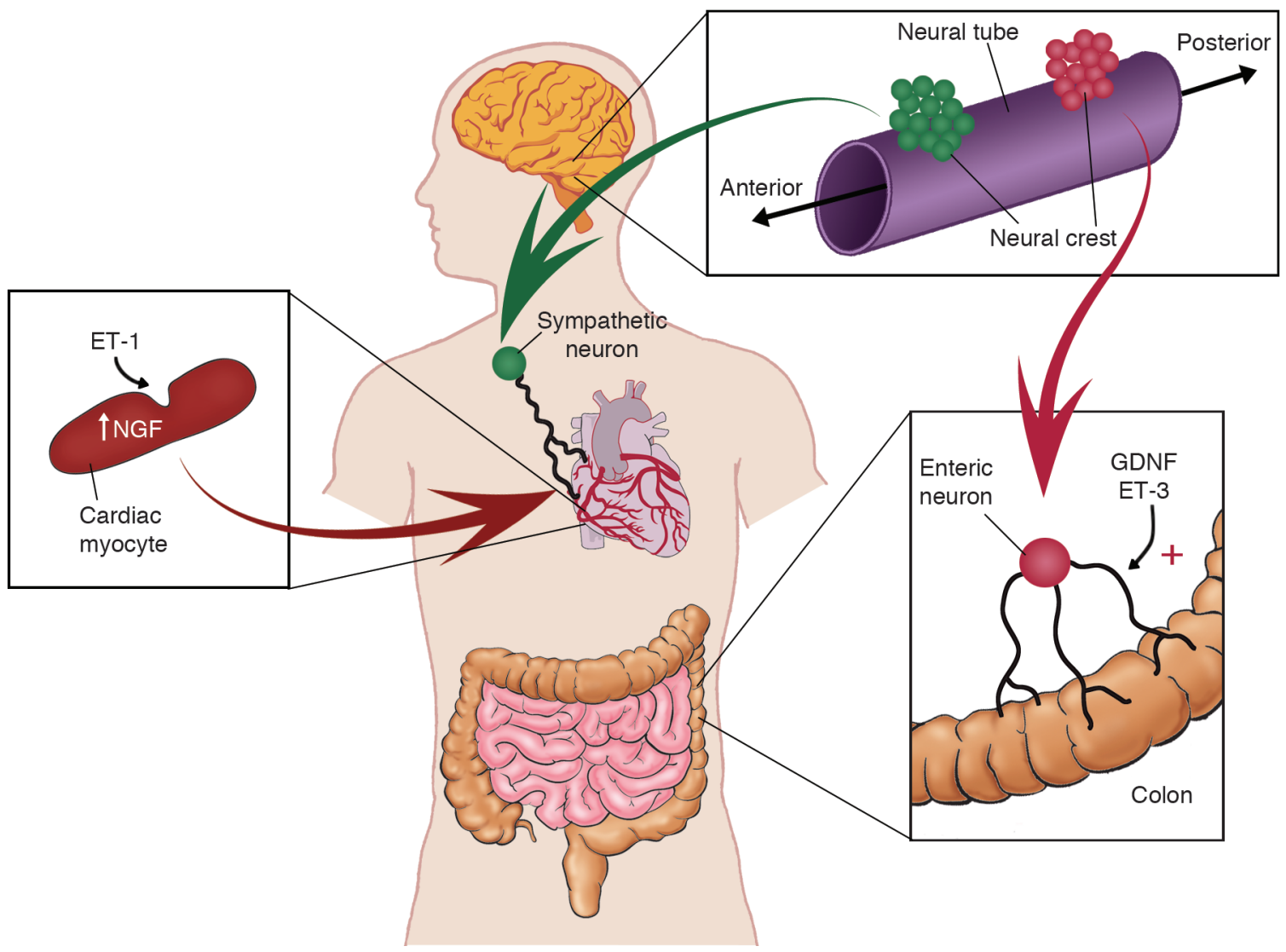

Figure 1

Distinctive pairing of endothelins and neurotrophic factors promotes target innervation during development. Although the sequential action of ET-1 to induce myocyte production of NGF is suggested to promote sympathetic innervation of the heart, more complex interactions of ET-3 and GDNF occur in the patterning of the enteric nervous system.

However, in comparison with the proposed sequential actions of ET-1 on cardiac myocytes to induce NGF for invading sympathetic fibers in the heart, two recent studies (13, 14) suggest that the interactions of ET- 3 and GDNF in the developing enteric nervous system are significantly more complex (Figure 1). Enteric neuron stem cells and differentiated progeny respond to both classes of ligand to mediate survival, differentiation, and target innervation, although sequential actions of ET-3 on gut mesenchyme to alter GDNF expression would require further evaluation. Nonetheless, these studies, together with the report by Ieda et al. (4), suggest that distinct endothelin-neurotrophic factor pairing may be coordinately utilized in different target organs during embryogenesis to regulate the complex process of target innervation.

\section{Potential roles of ET-1-NGF signaling in the adult heart}

Target-derived neurotrophins are critical for survival and fiber outgrowth during development, when they serve to match neuron numbers with target requirements. Why, then, are high levels of NGF (15) maintained in the adult heart? NGF can promote phenotypic changes in adult neurons, altering neuropeptide expression (16) and inducing enzymes that critically regulate neurotransmitter biosynthesis. A recent study by Birren and colleagues (17) provocatively suggests that, in addition to these long-term actions, neurotrophins may induce rapid switching from excitatory to inhibitory signals in sympathetic neurons that innervate the heart, effects that occur within minutes. Sympathetic neurons, grown in coculture with cardiac myocytes, synthesize both acetylcholine and norepinephrine in two distinct neurotransmitter pools. These neurotransmitters have opposing actions: acetylcholine is inhibitory, slowing the beat of myocytes, whereas norepinephrine is excitatory, depolarizing the cells and speeding their beat rate. Sympathetic neurons express the NGF receptor, TrkA, and the $\mathrm{p} 75$ receptor, which binds all neurotrophins, including BDNF.
Treatment of cocultures of sympathetic neurons and cardiac myocytes with NGF, to preferentially activate neuronal TrkA, promotes the release of norepinephrine and increases the myocyte beat rate fourfold. In contrast, treatment with BDNF activates neuronal $\mathrm{p} 75$, which induces the release of acetylcholine and significantly slows the rate of myocyte contraction. These observations suggest that the local secretion of neurotrophins - NGF from cardiac myocytes, and BDNF perhaps from the heart vasculature (18) - can rapidly exert opposing presynaptic actions to regulate heart rate and contractility.

How might these observations, and those of the current study (4), be used to modulate pathophysiologic responses of the heart? Following cardiac ischemic injury and reperfusion in rodents, both BDNF expression and NGF expression are dynamically regulated by myocytes and smooth muscle cells (19). Thus, strategies to impair NGF-mediated TrkA activation and promote BDNF-mediated p75 activation may prove beneficial in 
reducing sympathetic neuron sprouting and norepinephrine release, which in turn may prevent deleterious arrhythmic effects (20). In addition, the studies by Ieda may help to mechanistically dissect the complex actions of ET- 1 in congestive heart failure (4). In the adult, $\mathrm{ET}_{\mathrm{A}}$ receptors are expressed by VSMCs and cardiac myocytes, where vasoconstriction and proliferative actions predominate. $\mathrm{ET}_{\mathrm{B}}$ receptors on endothelial cells may in part counteract these effects by stimulating the release of nitric oxide and prostacyclin (5). In congestive heart failure, NGF levels in the heart decrease (21) as plasma norepinephrine levels rise; concomitantly, ET-1 plasma levels rise as well (22), although it is not clear how these changes are interrelated. Additionally, a recent report suggests that ET-1 overexpression in the adult mouse heart leads to a dilated cardiomyopathy and death (23). Given the developmental role of ET-1 in regulating NGF production by cardiac myocytes, evaluation of similar actions in the adult heart, and secondary effects on sympathetic function, may be warranted. Perhaps these lines of investigation will elucidate the complex interactions between myocyte and nerve in pathologic states, provide insight into the disappointing results of ET-1 blockade in congestive heart failure, and yield new pharmacologic targets for the future.

\section{Acknowledgments}

Barbara Hempstead is supported by grants from the National Institute of Neurological Disorders and Stroke, the National Heart, Lung, and Blood Institute, and the Burroughs Wellcome Fund.
Address correspondence to: Barbara L. Hempstead, Department of Medicine, Room C-606, Weill Medical College of Cornell University, 1300 York Avenue, New York, New York 10021, USA. Phone: (212) 746-6215; Fax: (212) 746-8866; E-mail: blhempst@med.cornell.edu.

1. Sofriniew, M.V., Howe, C.L., and Mobley, W.C. 2001. Nerve growth factor signaing, neuroprotection and neural repair. Annu. Rev. Neurosci. 24:1217-1281.

2. Patapoutian, A., Backus, C., Kispert, A., and Reichardt, L.F. 1999. Regulation of neurotrophin-3 expression by epithelial-mesenchymal interactions:the role of Wnt factors. Science. 283:1180-1183.

3. Hassankani, A., et al. 1995. Overexpression of NGF within the heart of transgenic mice causes hyperinnervation, cardiac enlargement and hyperplasia of ectopic cells. Dev. Biol. 169:309-321.

4. Ieda, M., et al. 2004. Endothelin-1 regulates cardiac sympathetic innervation in the rodent heart by controlling nerve growth factor expression. J. Clin. Invest. 113:876-884. doi:10.1172/JCI200419480.

5. Rich, S., and McLaughlin, V.V. 2003. Endothelin receptor blockers in cardiovascular disease. Circulation. 108:2184-2190.

6. Kanzawa, N., et al. 2002. Competency of embryonic cardiomyocytes to undergo Purkinje fiber differentiation is regulated by endothelin receptor expression. Development. 129:3185-3194.

7. Kurihara, Y., et al. 1995. Aortic arch malformations and ventricular septal defects in mice deficient in endothelin-1. J. Clin. Invest. 96:293-300.

8. Clouthier, D.E., et al. 1998. Cranial and cardiac neural crest defects in endothelin-A receptor deficient mice. Development. 125:813-824.

9. Thomas, T., et al. 1998. A signaling cascade involving endothelin-1, dHAND and $\mathrm{msx} 1$ regulates development of neural crest-derived branchial arch mesenchyme. Development. 125:3005-3014.

10. Glenbova, N.O., and Ginty, D.D. 2004. Heterogeneous requirement of NGF for sympathetic target innervation in vivo. J. Neurosci. 24:743-751.

11. Taraviras, S., and Pachnis, V. 1999. Development of the mammalian enteric nervous system. Curr. Opin. Genet. Dev. 9:321-327.
12. Chakravarti, A.L. 2001. Hirschprung's disease. In The Metabolic and Molecular Bases of Inherited Disease. C.R. Scriver et al., editors. McGraw-Hill. New York, New York, USA. 6231-6255.

13. Barlow, A., de Graaff, E., and Pachnis, V. 2003. Enteric nervous system progenitors are coordinately controlled by the $\mathrm{G}$ protein-coupled receptor EDNRB and the receptor tyrosine kinase RET. Neuron. 40:905-916.

14. Kruger, G.M., et al. 2003. Temporally distinct requirements for endothelin receptor $B$ in the generation and migration of gut neural crest stem cells. Neuron. 40:917-929.

15. MacGrogan, D., Saint-Andre, J.P., and Dicou, E. 1992. Expression of nerve growth factor and nerve growth factor receptor genes in human tissues and in prostatic adenocarcinoma cell lines. J. Neurochem. 59:1381-1391.

16. Shadiack, A.M., Sun, Y., and Zigmond, R.E. 2001. Nerve growth factor antiserum induces axotomy-like changes in neuropeptide expression in intact sympathetic and sensory neurons. J. Neurosci. 21:363-371.

17. Yang, B., Slonimsky, J.D., and Birren, S.J. 2002. A rapid switch in sympathetic neurotransmitter release properties mediated by the 75 receptor. Nat. Newrosci. 5:539-545.

18. Donovan, M.J., et al. 2000. Brain derived neurotrophic factor is an endothelial cell survival factor required for intramyocardial vessel stabilization. Development. 127:4531-4540.

19. Hiltunen, J.O., Launkainen, A., Vakeva, A., Meri, S., and Saarma, M. 2001. Nerve growth factor and brain-derived neurotrophic factor mRNAs are regulated in distinct cell populations of rat heart after ischaemia and reperfusion. J. Pathol. 194:247-253.

20. Cao, J.M., et al. 2000. Nerve sprouting and sudden cardiac death. Circ. Res. 86:816-821.

21. Kaye, D.M., Vaddadi, G., Gruskin, S.L., Du, X.J., and Esler, M.D. 2000. Reduced myocardial nerve growth factor expression in human and experimental heart failure. Circ. Res. 86:80-84.

22. Stewart, D.J., Cernacek, P., Costello, K.B., and Rouleau, J.L. 1992. Elevated endothelin-1 in heart failure and loss of normal response to postural change. Circulation. 85:510-517.

23. Yang, L.L., et al. 2004. Conditional cardiac overexpression of endothelin-1 induces inflammation and dilated cardiomyopathy in mice. Circulation. 109:255-261. 